\title{
Efficacy and safety of thrombolysis for acute ischemic stroke with atrial fibrillation: a meta-analysis
}

Yunzhen $\mathrm{Hu}^{1 * \dagger}$ and Chunmei $\mathrm{Ji}^{2+}$

\begin{abstract}
Background: The efficacy and safety of intravenous thrombolysis (IVT) for acute ischemic stroke with atrial fibrillation (AF) is still controversial.

Methods: We conducted a meta-analysis of all relevant studies, retrieved through systematic search of PubMed, Embase, and Cochrane databases up to December 31, 2019. Modified Rankin Scale (mRS) scores of 0-1 at 90 days, mRS of $0-2$ at 90 days, overall mortality, and incidence of symptomatic intracranial hemorrhage $(\mathrm{s} \mid \mathrm{CH})$ were collected as outcome measures. Fixed- and random-effects meta-analytical models were applied, and betweenstudy heterogeneity was assessed.
\end{abstract}

Results: A total of 8509 patients were enrolled in 18 studies. A comparison of IVT treatment in AF versus non-AF patients showed that AF was associated with a significantly lower proportion of patients with mRS of $0-1$ ( $24.1 \%$ vs. $34.5 \%$; OR 0.59; 95\% Cl 0.43-0.81; $P<0.001$ ), mRS of $0-2$ (33.6\% vs. 47.8\%; OR 0.55; 95\% Cl 0.43-0.70; $P<0.001$ ), as well as significantly higher mortality (19.4\% vs. 11.5\%; OR 2.05; 95\% Cl 1.79-2.36; $P<0.001)$ and higher incidence of sICH (6.4\% vs. 4.1\%; OR 1.60; 95\% Cl 1.27-2.01; $P<0.001)$. A comparison of AF patients who were subjected or not to IVT showed that thrombolysis carried a higher risk of sICH (5.7\% vs. 1.6\%; OR 3.44; $95 \% \mathrm{Cl} 2.04-5.82 ; P<0.001$ ) and was not associated with a better prognosis. Subgroup analysis in prospective studies also suggested a poorer functional prognosis and higher mortality in AF patients treated with IVT compared with those who did not receive IVT. Some heterogeneity was present in this meta-analysis.

Conclusions: Acute IS patients with AF had worse outcomes than those without AF after thrombolytic therapy, and had a higher incidence of sICH after thrombolysis than those without thrombolysis. Thrombolysis in ischemic stroke patients with AF should be carefully considered based on clinical factors such as NIHSS score, age, and the type of AF.

Keywords: Meta-analysis, Atrial fibrillation, Ischemic stroke, Thrombolysis

\footnotetext{
* Correspondence: 1504039@zju.edu.cn

${ }^{\dagger}$ Yunzhen $\mathrm{Hu}$ and Chunmei Ji contributed equally to this work.

${ }^{1}$ Department of Pharmacy, The First Affiliated Hospital, College of Medicine,

Zhejiang University, Hangzhou, China

Full list of author information is available at the end of the article
}

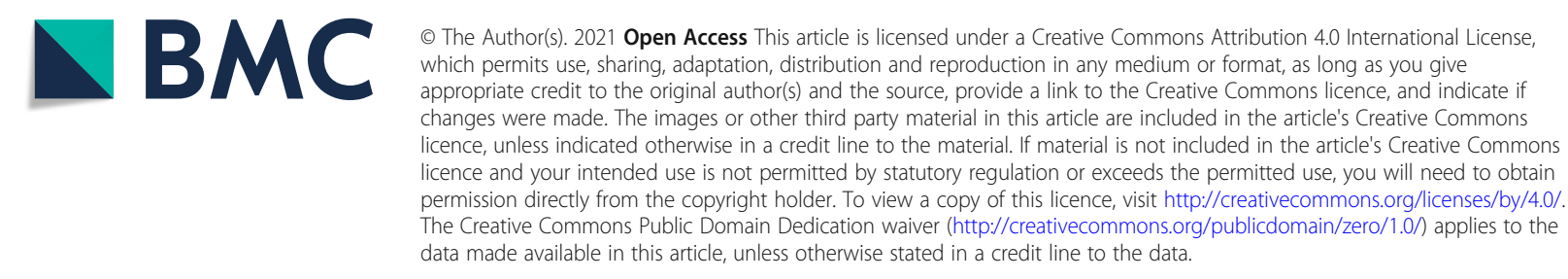




\section{Background}

Atrial fibrillation (AF) is a major risk factor for cardioembolic stroke, which is responsible for up to one-third of all ischemic stroke (IS) cases [1]. AF is associated with a 4-5-fold increased risk of IS [2], and AF-related strokes are more frequently fatal or disabling than those without a history of AF [3]. Intravenous thrombolysis (IVT) in acute IS patients within $4.5 \mathrm{~h}$ of onset can significantly improve functional outcome and reduce the risk of death and severe disability from stroke [4].

What is the efficacy and safety of thrombolysis for acute IS with AF? There are different opinions on this subject. When IS patients with and without AF are compared, most studies indicate that patients without AF show better 90-day functional outcomes after receiving thrombolytic therapy than those with AF [5-13]. However, a few studies reach the opposite conclusion [14, 15]. In addition, most studies show that AF patients pretreated with IVT have higher mortality [5-12, 15-19] and $\mathrm{sICH}$ incidence $[5,7-12,16,17,19]$ than those without AF. When comparing AF patients treated or not with IVT, some studies indicate that IVT is associated with much better functional outcomes [7, 9, 15, 20, 21]. However, several other studies reach the opposite conclusion $[5,11]$. In the case of AF patients treated with IVT therapy, five studies showed lower mortality [7, 9, $15,20,21]$ with respect to those who did not receive IVT therapy. On the contrary, two studies reported higher mortality $[5,11]$. Due to these controversial results, we performed a meta-analysis of all relevant studies measuring the efficacy and safety of thrombolytic therapy for acute IS patients with AF.

\section{Methods}

\section{Data sources and search strategy}

We used PubMed, Embase and Cochrane electronic databases to identify all published studies assessing the efficacy and safety of thrombolytic therapy for acute IS with AF, up to December 31, 2019. The search terms were ((stroke) OR (cerebrovascular disorders) OR (cerebral infarction) OR (brain infarction)) AND ((tissue plasminogen activator) OR (alteplase) OR (thrombolytic therapy) OR (thrombolysis)) AND ((atrial fibrillation) OR (AFib) OR (AF)). The search did not have any language restrictions.

\section{Study selection}

Two investigators (YZ Hu and $\mathrm{CM}$ Ji) independently performed the study selection. Studies were considered to be potentially eligible for this meta-analysis if they met the following criteria: (1) they compared the efficacy and safety of thrombolysis in AF versus non-AF patients; (2) they measured the efficacy and safety of IVT in AF patients and compared the outcome with AF patients not treated with IVT; (3) they included sufficient data on the modified Rankin Scale (mRS) $0-1$ and mRS 0-2, mortality and the incidence of symptomatic intracranial hemorrhage $(\mathrm{sICH})$. Exclusion criteria were as follows: (1) non-clinical studies, such as reviews, meta-analysis, case reports, letters, or comments; (2) no clinical outcome data.

\section{Quality assessment}

The quality of the cohort studies was assessed using the Newcastle-Ottawa Quality Assessment Scale (NOS) [22]. This scale is recommended by the Cochrane NonRandomized Studies Methods Working Group and consists of eight items that assess patient selection, study comparability and outcome. Studies with scores $0-3$ are considered of low quality, 4-6 of moderate quality, and 79 of high quality. The RCT study was assessed with the Cochrane collaboration tool for assessing risk of bias.

The potential sources of heterogeneity explored by the mete-regression analysis. The studies' characteristics were grouped as follows: the type of study design (prospective/ retrospective); stroke severity (NIHSS $<16 / \geq 16$ ), thrombolysis time window $(<3 \mathrm{~h} / \geq 3 \mathrm{~h})$ and the different online year of study. Meta-regression with random effects model was preferred with aggregate-level data.

\section{Data extraction and outcome measures}

Two investigators ( $\mathrm{YZ} \mathrm{Hu}$ and $\mathrm{CM} \mathrm{Ji}$ ) independently extracted data from the studies (authors, year of publication, design), population characteristics (number of patients, average age, sex ratio, presence of hypertension, diabetes mellitus and dyslipidemia, median baseline NIHSS score, onset to needle, mortality and incidence of sICH).

The primary efficacy endpoint was "excellent outcome" (mRS of 0-1, 90 days after stroke), and the secondary efficacy endpoint was "good outcome" (mRS of $0-2$, 90 days after stroke). The primary safety endpoint was mortality and the secondary safety endpoint was sICH incidence.

\section{Statistical analysis}

The proportion of patients with mRS of $0-1$ and $0-2$, the mortality and the sICH incidence were compared between AF IVT and non-AF IVT groups and (or) AF IVT versus AF non-IVT groups. We calculated the odds ratios (ORs) and corresponding 95\% confidence intervals (CIs) for each outcome. The heterogeneity of the studies included in our article was assessed by means of the $I^{2}$ test [23]. The $I^{2}$ ranged from 0 to $100 \% . I^{2}>50 \%$ indicated high heterogeneity and in these cases the randomeffects model was used for meta-analysis. Otherwise, the fixed-effects model was used. A funnel plot and Egger's test were used to assess publication bias in the metaanalysis. $P<0.05$ was considered statistically significant. 
All statistical tests were performed using STATA software (11.0; StataCorp, College Station, TX, USA).

\section{Results}

\section{Study characteristics}

We identified 1049 potentially relevant studies, but 1024 were excluded after screening the title and abstract. The full texts of the remaining 25 studies were retrieved for detailed evaluation. Based on the search criteria, a total of 18 studies reporting on the efficacy and safety of thrombolysis for acute IS with AF were included in this study (Fig. 1). The baseline characteristics of the patients included in these studies are shown in Tables 1 and 2 . Eleven studies [6, 8, 10, 12-14, 16-19, 24] compared IVT treatment in AF versus non-AF patients, two studies [20,21] compared AF patients treated or not with IVT, and five studies [5, 7, 9, 11, 15] included both comparisons.

\section{Outcome of thrombolysis in AF versus non-AF patients} In patients receiving thrombolytic therapy, significantly lower proportions of mRS $0-1(24.1 \%$ vs. $34.5 \%$; OR 0.59; 95\% CI $0.43-0.81 ; I^{2}=71.0 \% ; P<0.001$, Fig. 2a) and mRS $0-2$ (33.6\% vs. $47.8 \%$; OR 0.55 ; $95 \%$ CI $0.43-$ $0.70 ; I^{2}=55.2 \% ; P<0.001$, Fig. $\left.2 \mathrm{~b}\right)$ scores were seen in patients with AF when compared with patients without AF. On the contrary, significantly higher mortality (19.4\% vs. $11.5 \%$; OR 2.05 ; $95 \%$ CI $1.79-2.36 ; I^{2}=44.1 \%$; $P<0.001$, Fig. $2 \mathrm{c})$ and sICH incidence $(6.4 \%$ vs. $4.1 \%$;
OR 1.60; 95\% CI 1.27-2.01; $I^{2}=0.0 \% ; P<0.001$, Fig. $2 \mathrm{~d}$ ) were seen in $\mathrm{AF}$ patients.

\section{Comparing the outcome of AF patients treated or not with IVT}

In patients with $\mathrm{AF}$, there were no significant differences in the proportion of $\mathrm{mRS} 0-1$ (24.0\% vs. $21.4 \%$; OR 1.52 ; 95\% CI 0.83-2.79; $I^{2}=87.1 \% ; P=0.172$, Fig. 3a), mRS 0$2\left(31.0 \%\right.$ vs. $32.5 \%$; OR $1.37 ; 95 \%$ CI $0.72-2.60 ; I^{2}=$ $90.5 \% ; P=0.331$, Fig. $3 \mathrm{~b})$ scores or mortality $(22.4 \%$ vs. 20.7\%; OR 0.95; 95\% CI $0.63-1.44 ; I^{2}=71.7 \% ; P=0.813$, Fig. 3c) between those treated or not with IVT. In contrast, the incidence of sICH was significantly higher in patients treated with IVT therapy $(5.7 \%$ vs. $1.6 \%$; OR 3.44; 95\% CI 2.04-5.82; $I^{2}=0.0 \% ; P<0.001$ ) (Fig. 3d).

\section{Subgroup analysis and meta-regression analysis}

Subgroup analysis was performed according to the study design (Table 3). In both prospective and retrospective AF IVT versus non-AF IVT studies, the functional outcome of patients with AF treated with IVT was worse $(P<0.001)$ than in patients without AF, and the mortality $(P<0.001)$ and $\mathrm{sICH}$ incidence $(P<0.01)$ were also higher. On the other hand, in AF IVT versus AF nonIVT prospective studies, the results suggested a poorer functional prognosis $(P<0.01)$ and higher mortality $(P<$ $0.05)$ in AF patients treated with thrombolytic therapy than in those who did not receive it, and in retrospective studies, there was a higher sICH incidence in AF IVT patients $(P<0.001)$.

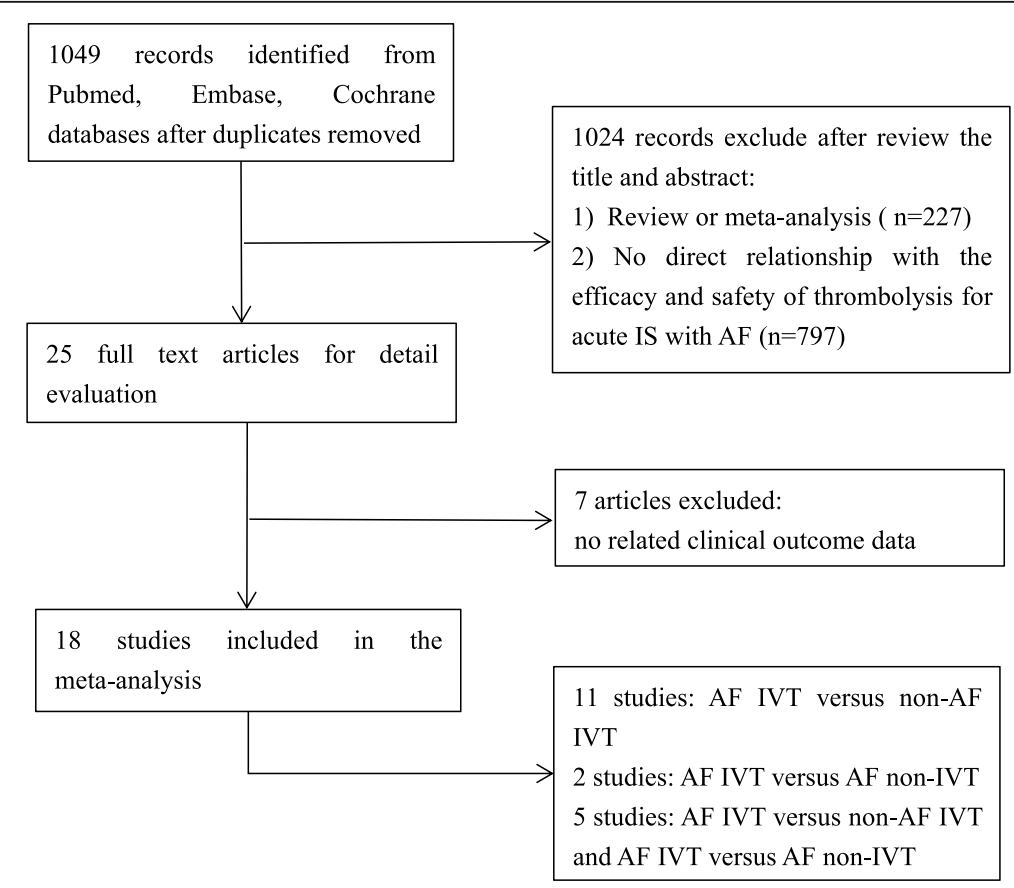

Fig. 1 Literature search profile 


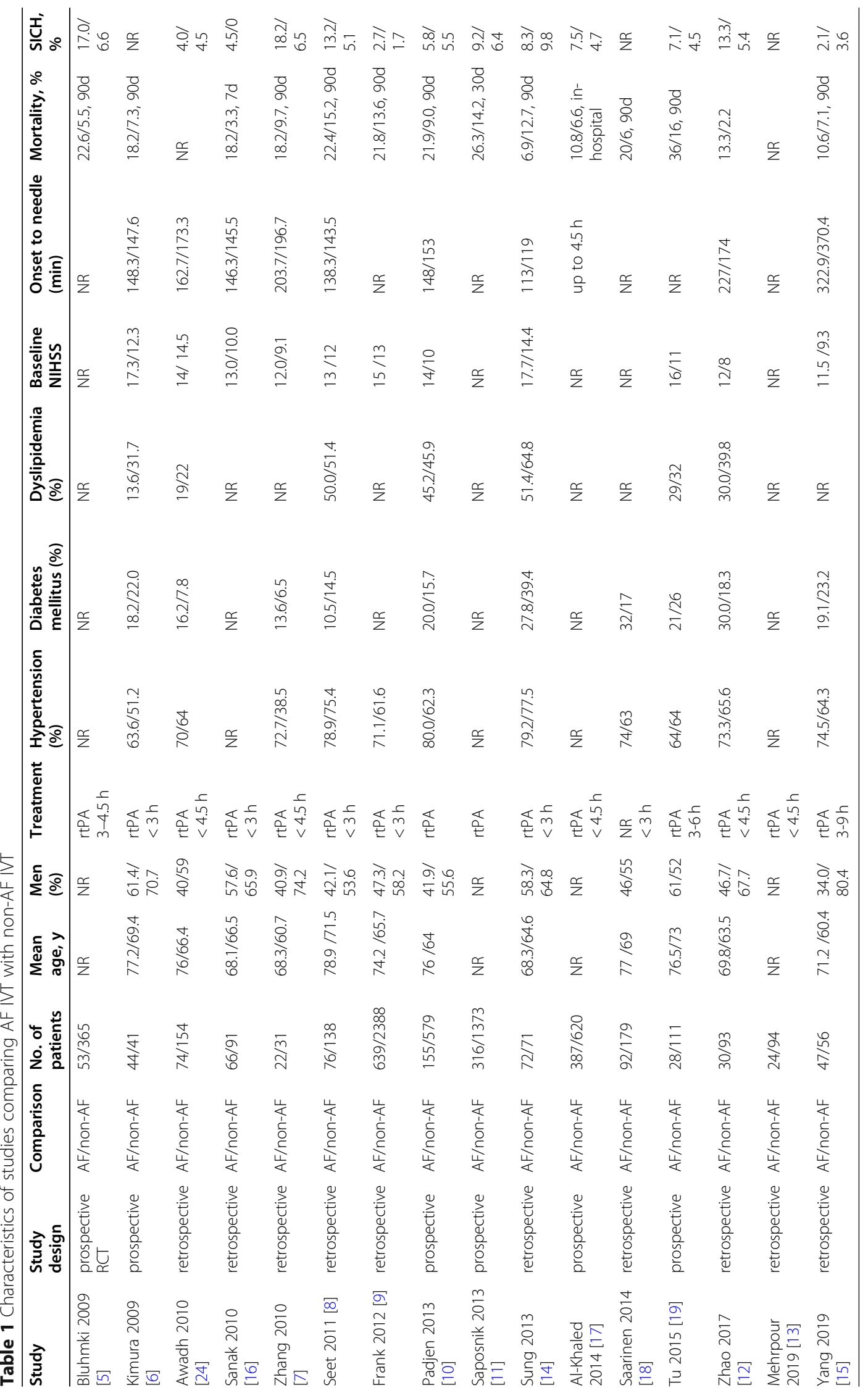




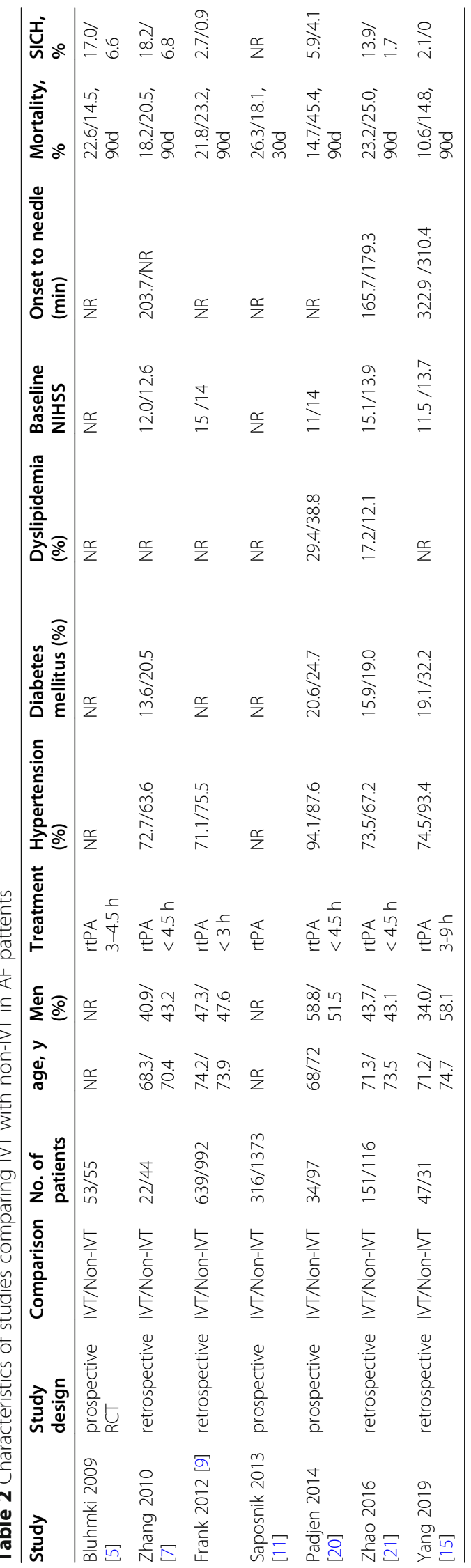



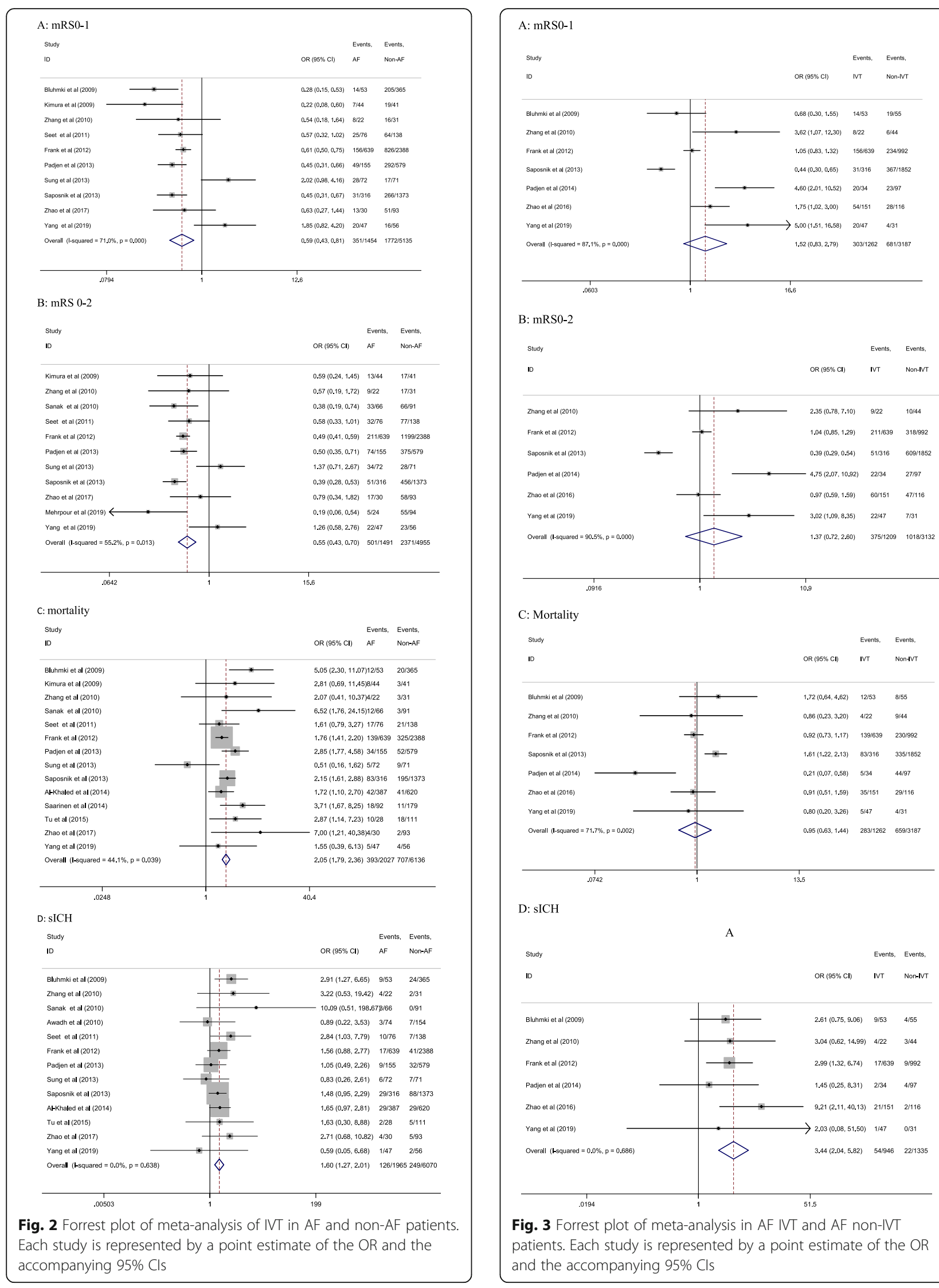

B: mRS0-2

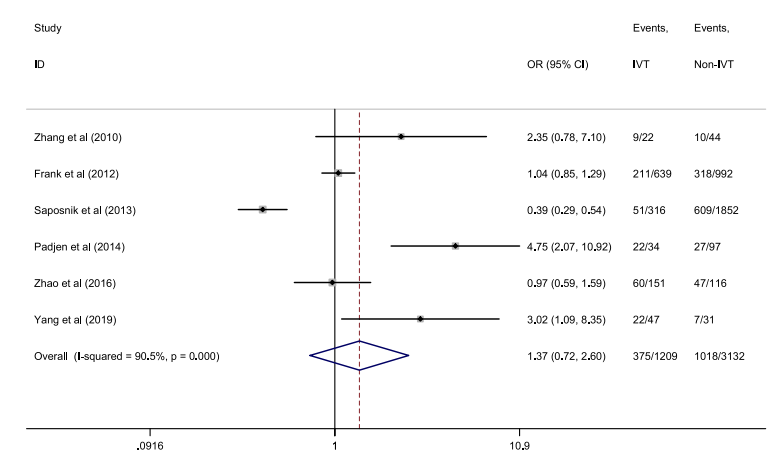

C: Mortality

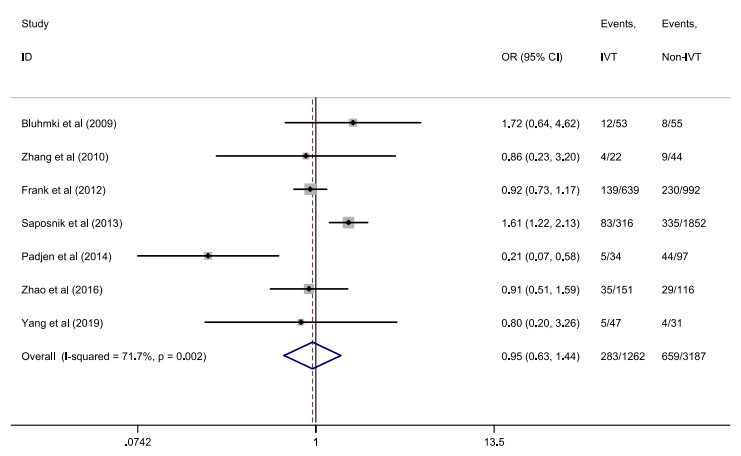

D: $\mathrm{sICH}$

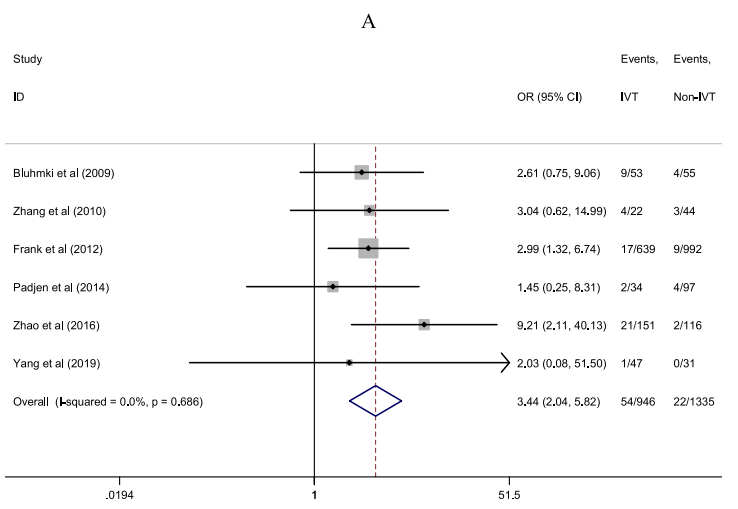

Fig. 3 Forrest plot of meta-analysis in AF IVT and AF non-IVT patients. Each study is represented by a point estimate of the OR and the accompanying $95 \% \mathrm{Cls}$ 
Table 3 Subgroup analyses of efficacy and safety of thrombolysis for acute IS with AF based on study design

\begin{tabular}{|c|c|c|c|c|c|c|}
\hline \multirow[t]{2}{*}{ Study design } & \multicolumn{2}{|l|}{ mRS 0-1 } & \multicolumn{2}{|c|}{ Test of association } & \multicolumn{2}{|c|}{ Heterogeneity } \\
\hline & $\overline{\mathrm{AF}}$ & Non-AF & OR $(95 \% \mathrm{Cl})$ & $P$ & $\overline{I^{2}(\%)}$ & $P$ \\
\hline Retrospective $(n=6)$ & $250 / 886$ & $990 / 2777$ & $0.68(0.57-0.81)$ & $<0.001$ & 75.0 & 0.006 \\
\hline \multirow[t]{2}{*}{$\operatorname{Prospective}(n=4)$} & $101 / 568$ & $782 / 2358$ & $0.41(0.32-0.52)$ & $<0.001$ & 0.0 & 0.343 \\
\hline & IVT & Non-IVT & OR $(95 \% \mathrm{Cl})$ & $P$ & $I^{2}(\%)$ & $P$ \\
\hline Retrospective $(n=4)$ & $238 / 859$ & $272 / 1183$ & $1.24(1.01-1.52)$ & 0.039 & 74.5 & 0.008 \\
\hline \multirow[t]{3}{*}{$\operatorname{Prospective}(n=3)$} & $65 / 403$ & $409 / 2004$ & $0.65(0.48-0.87)$ & 0.005 & 92.1 & $<0.001$ \\
\hline & \multicolumn{2}{|c|}{ mRS 0-2 } & \multicolumn{2}{|c|}{ Test of association } & \multicolumn{2}{|c|}{ Heterogeneity } \\
\hline & AF & Non-AF & OR $(95 \% \mathrm{Cl})$ & $P$ & $I^{2}(\%)$ & $P$ \\
\hline Retrospective $(n=8)$ & $363 / 976$ & $1523 / 2962$ & $0.53(0.46-0.62)$ & $<0.001$ & 63.2 & 0.008 \\
\hline \multirow[t]{2}{*}{ Prospective $(n=3)$} & $138 / 515$ & $848 / 1993$ & $0.44(0.35-0.55)$ & $<0.001$ & 0 & 0.474 \\
\hline & IVT & Non-IVT & OR (95\% Cl) & $P$ & $I^{2}(\%)$ & $P$ \\
\hline Retrospective $(n=4)$ & $302 / 859$ & $382 / 1183$ & $1.10(0.91-1.33)$ & 0.317 & 50.6 & 0.108 \\
\hline \multirow[t]{3}{*}{$\operatorname{Prospective}(n=2)$} & $73 / 350$ & $636 / 1949$ & $0.53(0.40-0.70)$ & $<0.001$ & 96.7 & $<0.001$ \\
\hline & \multicolumn{2}{|c|}{ Mortality } & \multicolumn{2}{|c|}{ Test of association } & \multicolumn{2}{|c|}{ Heterogeneity } \\
\hline & AF & Non-AF & OR $(95 \% \mathrm{Cl})$ & $P$ & $I^{2}(\%)$ & $P$ \\
\hline Retrospective $(n=8)$ & $204 / 1044$ & $378 / 3047$ & $1.86(1.54-2.25)$ & $<0.001$ & 49.7 & 0.053 \\
\hline \multirow[t]{2}{*}{ Prospective $(n=6)$} & 189/983 & $329 / 3089$ & $2.30(1.88-2.82)$ & $<0.001$ & 25.7 & 0.242 \\
\hline & IVT & Non-IVT & OR $(95 \% \mathrm{Cl})$ & $P$ & $I^{2}(\%)$ & $P$ \\
\hline Retrospective $(n=4)$ & $183 / 859$ & $272 / 1183$ & $0.91(0.74-1.13)$ & 0.413 & 0.0 & 0.998 \\
\hline \multirow[t]{3}{*}{ Prospective $(n=3)$} & $100 / 403$ & $387 / 2004$ & $1.34(1.04-1.73)$ & 0.025 & 86.3 & 0.001 \\
\hline & \multicolumn{2}{|c|}{ sICH } & \multicolumn{2}{|c|}{ Test of association } & \multicolumn{2}{|c|}{ Heterogeneity } \\
\hline & AF & Non-AF & OR $(95 \% \mathrm{Cl})$ & $P$ & $I^{2}(\%)$ & $P$ \\
\hline Retrospective $(n=8)$ & $48 / 1026$ & $71 / 3022$ & $1.67(1.14-2.46)$ & 0.009 & 0 & 0.490 \\
\hline \multirow[t]{2}{*}{$\operatorname{Prospective}(n=5)$} & $78 / 939$ & $178 / 3048$ & $1.56(1.17-2.07)$ & 0.002 & 0 & 0.509 \\
\hline & IVT & Non-IVT & OR $(95 \% \mathrm{Cl})$ & $P$ & $I^{2}(\%)$ & $P$ \\
\hline Retrospective $(n=4)$ & $43 / 857$ & $14 / 1183$ & $4.04(2.16-7.55)$ & $<0.001$ & 0.0 & 0.566 \\
\hline Prospective $(n=2)$ & $11 / 87$ & $8 / 152$ & $2.18(0.80-5.88)$ & 0.126 & 0.0 & 0.592 \\
\hline
\end{tabular}

The meta-regression analysis explained the variability between studies based on the mRS $0-1$ in is the thrombolysis in AF versus non-AF patients. The results showed as the following: the different online year of studies $55.75 \%$, type of study design $59.01 \%$, and multivariable adjusted $\mathrm{R}^{2}$ was $84.37 \%$ (Table 4).

\section{Assessment of quality and publication bias}

Most included cohort studies were of high quality, with NOS scores ranging from 6 to 9 (Table 5). The mean NOS for all included studies was 7 and the RCT (Bluhmki et al. 2009) assessed with the Cochrane collaboration tool showed a low risk of bias. The study was conducted blindly, with random sequence generation and allocation concealment. The outcome data in the included RCT study was subjective. A funnel plot and Egger's test were performed to evaluate publication bias in this metaanalysis. Egger's test $(P=0.783)$ showed there was no
Table 4 Meta-regression according to methodological covariates of AF patients treated or not with IVT

\begin{tabular}{lllll}
\hline mRS0-1 & & & & \\
\hline Variable & $\begin{array}{l}\text { Number of } \\
\text { estimates }\end{array}$ & Tau 2 & $\mathbf{R}^{\mathbf{2}}$ & $\begin{array}{l}\boldsymbol{P} \text { - } \\
\text { value }\end{array}$ \\
\hline Year & 10 & 0.1322 & $55.75 \%$ & 0.037 \\
Study design & 10 & 0.1367 & $59.01 \%$ & 0.033 \\
Stroke severity & 8 & 0.3839 & - & 0.848 \\
Thrombolysis time & 8 & 0.5316 & - & 0.975 \\
window & & & & \\
Multivariable adjusted $R^{2}$ of the models 84.37\% & & & \\
\hline
\end{tabular}

Tau2 variance residual variation between-study due to heterogeneity, $R^{2}$ Adjuested $\mathrm{R}^{2}$ of the Meta-regression model 
Table 5 Quality assessments of the included studies with the NOS

\begin{tabular}{|c|c|c|c|c|}
\hline Study & Selection & Comparability & Outcome & Total score \\
\hline Mehrpour et al. 2019 [13] & $* * * *$ & * & $* *$ & 7 \\
\hline Yang et al. 2019 [15] & $* * * *$ & * & $* * *$ & 8 \\
\hline Zhao et al. 2017 [12] & $* * *$ & $* *$ & $* *$ & 7 \\
\hline Zhao et al. 2016 [21] & $* * *$ & $* *$ & $* *$ & 8 \\
\hline Tu et al. 2015 [19] & $* * *$ & $* *$ & $* *$ & 7 \\
\hline Al-khaled et al. 2014 [17] & $* * *$ & * & * & 5 \\
\hline Saarinen et al. 2014 [18] & $* * *$ & $* *$ & $* * *$ & 8 \\
\hline Padjen et al. 2014 [20] & $* * *$ & $* *$ & $* *$ & 7 \\
\hline Padjen et la 2013 [10] & $* * * *$ & * & $* * *$ & 8 \\
\hline Saposnik et al. 2013 [11] & $* * * *$ & * & * & 6 \\
\hline Sung et al. 2013 [14] & $* * *$ & $* *$ & $* *$ & 7 \\
\hline Frank et al. 2012 [9] & $* * *$ & * & $* *$ & 6 \\
\hline Seet et al. 2011 [8] & $* * *$ & $* *$ & $* *$ & 7 \\
\hline Awadh et al. 2010 [25] & $* * * *$ & * & $* *$ & 7 \\
\hline Sanak et al. 2010 [16] & $* * * *$ & $* *$ & * & 7 \\
\hline Zhang et al. 2010 [7] & $* * *$ & $* *$ & $* *$ & 7 \\
\hline Bluhmki et al. 2009 [5] & $* * * *$ & $* *$ & $* * *$ & 9 \\
\hline Kimura et al. 2009 [6] & $* * *$ & * & $* *$ & 6 \\
\hline
\end{tabular}

significant evidence of publication bias. On the other hand, no significant publication bias was detected based on Begg's funnel plot (Fig. 4).

\section{Discussion}

Patients with stroke and AF have poorer neurological outcomes than those without AF [25]. AF appears to be an independent risk factor for in-hospital mortality, length of hospital stay, and increased treatment costs in stroke patients [26]. Kimura et al. reported that AF was independently associated with no early recanalization after IVT in acute IS [27]. A meta-analysis done by Yue et al. in 2016 showed that AF was associated with poor outcomes in thrombolyzed patients with acute IS [28]. With increasing number of studies reporting on the effects of thrombolysis in AF patients, we collected more evidence to compare the outcome of

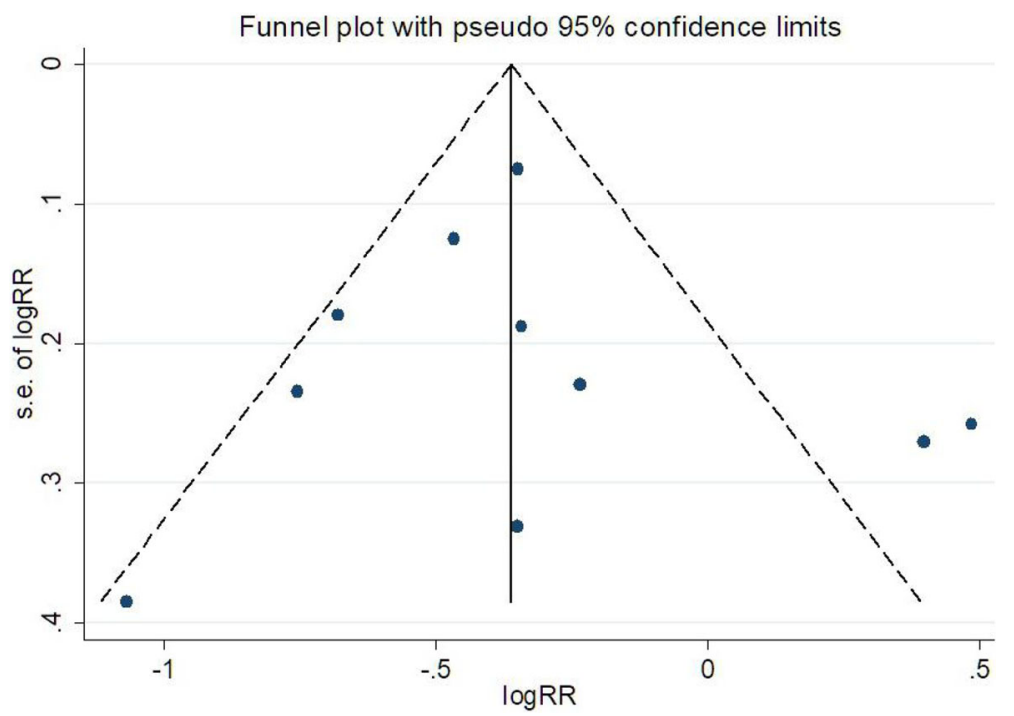

Fig. 4 Funnel plot of publication bias in the meta-analysis. The largest studies are plotted near the average, and smaller studies are spread evenly on both sides of the average, creating a roughly funnel-shaped distribution. Deviation from this shape can indicate publication bias 
thrombolytic therapy in acute IS patients with or without $\mathrm{AF}$, as well as of thrombolytic versus nonthrombolytic therapy in patients with AF. This allowed us to reach more comprehensive conclusions regarding the safety and efficacy of thrombolysis in acute IS patients with AF.

The final meta-analysis included 18 studies. Sixteen studies compared thrombolytic outcomes in acute IS patients with or without AF. These studies included 8509 patients (24.97\% of them with AF). Seven studies compared the outcome in AF patients treated or not with IVT. These studies included 4449 patients, $28.36 \%$ of whom received thrombolytic therapy. Five studies included both comparisons. Comparison of AF IVT versus non-AF IVT groups showed that AF was associated with a significantly lower proportion of patients with mRS of $0-1, \mathrm{mRS}$ of $0-290$ days after stroke and significantly higher mortality and $\mathrm{sICH}$ incidence. Comparison of AF IVT versus AF non-IVT groups showed that thrombolysis carried a higher risk of sICH and was not associated with a better prognosis. Subgroup analysis in prospective studies also suggested a poorer functional prognosis and higher mortality in AF patients treated with IVT when compared with those who did not receive IVT.

The explanation for the observed results are as follows: First, patients with AF have greater infarct sizes and worse collateral circulation, resulting in worse baseline symptoms $[15,16]$. Second, patients with AF appear to be more likely to have large or old thrombi, which are resistant to IVT [6]. In most of the included studies, baseline NIHSS was higher in the AF group than in the non-AF group. In addition, some $\mathrm{AF}$ patients were treated with anticoagulant therapy prior to IVT, which also increases the risk of bleeding. A meta-analysis showed that the risk of sICH after thrombolytic therapy was higher in patients receiving warfarin with subtherapeutic INR levels [29]. How to improve the efficacy and safety of thrombolysis in patients with AF is worthy of further studies. New thrombolytic drugs [30], telethrombolysis [31] and combination with mechanical thrombectomy [32] may offer new options.

Heterogeneity was present in several statistical results of our study. Potential confounders between the groups were not balanced during comparative analysis, which may be a source of heterogeneity. In the included studies, patients with acute IS and AF were generally older and had a higher NIHSS, but we did not adjust for age or NIHSS in the meta-analysis. For example, in the comparative analysis of $\mathrm{mRS} 0-1$ proportions between the AF-IVT and AF non-IVT groups, the $I^{2}$ was $71.0 \%$. Based on sensitivity analysis, heterogeneity was derived from two studies $[14,15]$. The causes of heterogeneity in these two studies were analyzed: one study [14] included more severe stroke (NIHSS > 10) patients, while the other study [15] included patients with a longer thrombolysis time window $(3-9 \mathrm{~h})$ than the others. Excluding these two studies did not change the statistical results, but the heterogeneity improved greatly (OR 0.52 ; 95\% CI $0.45-$ $\left.0.60 ; I^{2}=30.6 \% ; P<0.001\right)$. In addition, the specific type of AF also explained the heterogeneity. Seet et al. reported that patients with chronic AF had worse outcomes than non-AF patients, and was greater in patients with AF of longer duration [8]. Meanwhile the metaregression analysis showed that the heterogeneity may related to the different online year of studies and type of study design.

We acknowledge that our study has several limitations. First, there was only one RCT study (out of 18 studies). Second, sample sizes in most studies were relatively small and varied between groups, which may limit the analytical capacity. Third, in the included studies, the IVT time window was not uniform: in some cases it was less than $3 \mathrm{~h}$, in others less than $4.5 \mathrm{~h}$, and in one case 3-9 h. Fourth, whether intravenous thrombolysis was combined with mechanical thrombectomy was not mentioned in the included studies. Finally, individual data were insufficiently detailed to identify subgroups according to age range, baseline NIHSS score, onset to needle, type of AF and other clinical factors that may influence the efficacy and safety of thrombolysis in AF patients. Further studies with a double-blind design, larger sample sizes and well-matched patient characteristics should be considered.

\section{Conclusions}

Acute IS patients with AF had worse outcomes than those without $\mathrm{AF}$ after thrombolytic therapy as well as a higher incidence of sICH after thrombolysis than those without thrombolysis. Thrombolysis in IS patients with AF should be carefully considered based on clinical factors such as NIHSS score, age, whether patients are taking anticoagulant drugs and type of AF.

\section{Abbreviations}

IS: Ischemic stroke; AF: Atrial fibrillation; IVT: Intravenous thrombolysis; mRS: Modified Rankin Scale; sICH: symptomatic intracranial hemorrhage

\section{Acknowledgements}

Not applicable.

\section{Authors' contributions}

YZ Hu conceived and designed this study. YZ Hu and CM Ji contributed the literature search, study selection and data extraction. YZ Hu performed data analysis and drafted the manuscript. All authors reviewed and approved the final version of the manuscript.

\section{Funding}

This work was supported by the National Natural Science Foundation of China (81703612).

Availability of data and materials

All data generated or analyzed during this study are included in this publication. 


\section{Ethics approval and consent to participate}

Not applicable.

\section{Consent for publication}

Not applicable.

\section{Competing interests}

The authors declare that they have no competing interests.

\section{Author details}

'Department of Pharmacy, The First Affiliated Hospital, College of Medicine, Zhejiang University, Hangzhou, China. ${ }^{2}$ Research Division of Clinical Pharmacology, the First Affiliated Hospital of Nanjing Medical University, Nanjing, Jiangsu, China.

Received: 12 April 2020 Accepted: 4 February 2021 Published online: 11 February 2021

\section{References}

1. Schnabel RB, Haeusler KG, Healey JS, Freedman B, Boriani G, et al. Searching for atrial fibrillation Poststroke: a white paper of the AF-SCREEN international collaboration. Circulation. 2019;140(22):1834-50. https://doi.org/10.1161/ CIRCULATIONAHA.119.040267.

2. Wolf PA, Abbott RD, Kannel WB. Atrial fibrillation as an independent risk factor for stroke: the Framingham study. Stroke. 1991;22:983-8.

3. Freedman B, Potpara TS, Lip GY. Stroke prevention in atrial fibrillation. Lancet. 2016;388:806-17. https://doi.org/10.1016/S0140-6736(16)31257-0.

4. Powers WJ, Rabinstein AA, Ackerson T, Adeoye OM, Bambakidis NC, et al. 2018 Guidelines for the early Management of Patients with Acute Ischemic Stroke: a guideline for healthcare professionals from the American Heart Association/American Stroke Association. Stroke. 2018;9(3):e46-e110. https:// doi.org/10.1161/STR.0000000000000158.

5. Bluhmki E, Chamorro A, Dávalos A, Machnig T, Sauce C, Wahlgren N, Wardlaw J, Hacke W. Stroke treatment with alteplase given 3.0-4.5 h after onset of acute ischaemic stroke (ECASS III): additional outcomes and subgroup analysis of a randomised controlled trial. Lancet Neurol. 2009;8(12):1095-102. https://doi.org/10.1016/S1474-4422(09)702 64-9.

6. Kimura K, Iguchi Y, Shibazaki K, Iwanaga T, Yamashita S, Aoki J. IV t-PA therapy in acute stroke patients with atrial fibrillation. J Neurol Sci. 2009; 276(1-2):6-8. https://doi.org/10.1016/j.jns.2008.10.018.

7. Zhang JB, Ding ZY, Yang Y, Sun W, Hai F, Sui XN, Li XY, Wang HZ, Wang XT, Zheng JL. Thrombolysis with alteplase for acute ischemic stroke patients with atrial fibrillation. Neurol Res. 2010;32(4):353-8. https://doi.org/10.1179/ $016164110 \times 12656393665206$

8. Seet RC, Zhang Y, Wijdicks EF, Rabinstein AA. Relationship between chronic atrial fibrillation and worse outcomes in stroke patients after intravenous thrombolysis. Arch Neurol. 2011;68(11):1454-8. https://doi.org/10.1001/a rchneurol.2011.248.

9. Frank B, Fulton R, Weimar C, Shuaib A, Lees KR, VISTA Collaborators. Impact of atrial fibrillation on outcome in thrombolyzed patients with stroke: evidence from the virtual international stroke trials archive (VISTA). Stroke. 2012;43(7):1872-7. https://doi.org/10.1161/STROKEAHA.112.650838.

10. Padjen V, Bodenant M, Jovanovic DR, Ponchelle-Dequatre N, Novakovic N, Cordonnier C, Beslac-Bumbasirevic L, Leys D. Outcome of patients with atrial fibrillation after intravenous thrombolysis for cerebral ischaemia. J Neurol. 2013;260(12):3049-54. https://doi.org/10.1007/s0041 5-013-7119-4

11. Saposnik G, Gladstone D, Raptis R, Zhou L, Hart RG, Investigators of the Registry of the Canadian Stroke Network (RCSN) and the Stroke Outcomes Research Canada (SORCan) Working Group. Atrial fibrillation in ischemic stroke: predicting response to thrombolysis and clinical outcomes. Stroke. 2013;44(1):99-104. https://doi.org/10.1161/STROKEA HA.112.676551.

12. Zhao Q, Shan W, Liu L, Fu X, Liu P, Hu Y. Predictors of functional outcome and hemorrhagic complications in acute ischemic stroke patients treated with intravenous thrombolysis-a retrospective analysis. Int J Clin Pharmacol Ther. 2017;55(12):893-900. https://doi.org/10.5414/CP203117.

13. Mehrpour M, Afrakhte M, Shojaei SF, Sohrabi A, Ashayeri R, Esmaeili S, Bahadori M. Factors predicting the outcome of intravenous thrombolysis in stroke patients before rt-PA administration. Caspian J Intern Med. 2019;10(4): 424-30. https://doi.org/10.22088/cjim.10.4.424.

14. Sung SF, Chen YW, Tseng MC, Ong CT, Lin HJ. Atrial fibrillation predicts good functional outcome following intravenous tissue plasminogen activator in patients with severe stroke. Clin Neurol Neurosurg. 2013;115(7): 892-5. https://doi.org/10.1016/j.clineuro.2012.08.034.

15. Yang Y, Sun W, Li D, Li XY, Wang XT, Li SC, Zhao HJ, Zhang JB. Multimode computed-tomography-guided thrombolysis under a prolonged time window in acute ischemic stroke patients with atrial fibrillation. Int Heart J. 2019;60(4):822-9. https://doi.org/10.1536/ihj.18-636.

16. Sanák D, Herzig R, Král M, Bártková A, Zapletalová J, Hutyra M, Skoloudík D, Vlachová I, Veverka T, Horák D, Kanovský P. Is atrial fibrillation associated with poor outcome after thrombolysis? J Neurol. 2010;257(6):999-1003. https://doi.org/10.1007/s00415-010-5452-4.

17. Al-Khaled M, Matthis C, Eggers J. Predictors of in-hospital mortality and the risk of symptomatic intracerebral hemorrhage after thrombolytic therapy with recombinant tissue plasminogen activator in acute ischemic stroke. J Stroke Cerebrovasc Dis. 2014;23(1):7-11. https://doi.org/10.1016/j. jstrokecerebrovasdis.2012.04.004.

18. Saarinen JT, Rusanen $H$, Sillanpää $N$, Huhtala $H$, Numminen $H$, Elovaara I. Impact of atrial fibrillation and inadequate antithrombotic management on mortality in acute neurovascular syndrome. J Stroke Cerebrovasc Dis. 2014;23(9):2256-64. https://doi.org/10.1016/j. jstrokecerebrovasdis.2014.04.017.

19. Tu HT, Campbell BC, Christensen S, Desmond PM, De Silva DA, Parsons MW, Churilov L, Lansberg MG, Mlynash M, Olivot JM, Straka M, Bammer R, Albers GW, Donnan GA, Davis SM, EPITHET-DEFUSE Investigators. Worse stroke outcome in atrial fibrillation is explained by more severe hypoperfusion, infarct growth, and hemorrhagic transformation. Int J Stroke. 2015;10(4): 534-40. https://doi.org/10.1111/ijs.12007.

20. Padjen V, Jovanovic D, Berisavac I, Ercegovac M, Stefanovic Budimkic M, Stanarcevic P, Beslac BL. Effect of intravenous thrombolysis on stroke associated with atrial fibrillation. J Stroke Cerebrovasc. 2014;23(8):2199-205. https://doi.org/10.1016/j.jstrokecerebrovasdis.2014.04.035.

21. Zhao Q, Li X, Dong W, Ye M, Cao Y, Zhang M, Cheng Q, Zhou J, Chen G, Yu M, Hong S, Wei X, Wang B, Cui G, Zhang P, Ding H, Xu R, Chen Y, Xu Y. Factors associated with thrombolysis outcome in ischemic stroke patients with atrial fibrillation. Neurosci Bull. 2016;32(2):145-52. https://doi.org/10.1 007/s12264-016-0019-8.

22. Wells G, Shea B, O'Connell D, Peterson J, Welch V, Losos M. The NewcastleOttawa Scale (NOS) for assessing the quality of nonrandomised studies in Meta-analyses. Availabe at: http://www.ohri.ca/programs/clinical_ epidemiology/oxford.htm. 2003.

23. Higgins JP, Thompson SG. Quantifying heterogeneity in a meta-analysis. Stat Med. 2002;21(11):1539-58. https://doi.org/10.1002/sim.1186.

24. Awadh M, MacDougall N, Santosh C, Teasdale E, Baird T, Muir KW. Early recurrent ischemic stroke complicating intravenous thrombolysis for stroke: incidence and association with atrial fibrillation. Stroke. 2010;41(9):1990-5. https://doi.org/10.1161/STROKEAHA.109.569459.

25. Findler M, Molad J, Bornstein NM, Auriel E. Worse outcome in patients with acute stroke and atrial fibrillation following thrombolysis. Isr Med Assoc J. 2017;19(5):293-5.

26. Pan X, Simon TA, Hamilton M, Kuznik A. Comparison of costs and discharge outcomes for patients hospitalized for ischemic or hemorrhagic stroke with or without atrial fibrillation in the United States. J Thromb Thrombolysis. 2015;39(4):508-15. https://doi.org/10.1007/s11239-014-1144-8.

27. Kimura K, Iguchi Y, Yamashita S, Shibazaki K, Kobayashi K, Inoue T. Atrial fibrillation as an independent predictor for no early recanalization after IV-tPA in acute ischemic stroke. J Neurol Sci. 2008;267(1-2):57-61.

28. Yue R, Li D, Yu J, Li S, Ma Y, Huang S, Zeng Z, Zeng R, Sun X. Atrial fibrillation is associated with poor outcomes in Thrombolyzed patients with acute ischemic stroke: a systematic review and meta-analysis. Medicine (Baltimore). 2016;95(10):e3054. https://doi.org/10.1097/MD. 0000000000003054

29. Miedema I, Luijckx GJ, De Keyser J, Koch M, Uyttenboogaart M. Thrombolytic therapy for ischaemic stroke in patients using warfarin: a systematic review and meta-analysis. J Neurol Neurosurg Psychiatry. 2012; 83(5):537-40. https://doi.org/10.1136/jnnp-2011-301794.

30. Elmaraezy A, Abushouk Al, Saad S, Eltoomy M, Mahmoud O, Hassan HM, Aboelmakarem A, Fotoh AA, Althaher F, Huy NT, Hirayama K. Desmoteplase for acute ischemic stroke: a systematic review and Metaanalysis of 
randomized controlled trials. CNS Neurol Disord Drug Targets. 2017;16(7): 789-99. https://doi.org/10.2174/1871527315666161213110104.

31. Baratloo A, Rahimpour L, Abushouk Al, Safari S, Lee CW, Abdalvand A.

Effects of Telestroke on thrombolysis times and outcomes: a meta-analysis. Prehosp Emerg Care. 2018;22(4):472-84. https://doi.org/10.1080/10903127.2 017.1408728.

32. Mistry EA, Mistry AM, Nakawah MO, Chitale RV, James RF, Volpi JJ, Fusco MR. Mechanical Thrombectomy outcomes with and without intravenous thrombolysis in stroke patients: a meta-analysis. Stroke. 2017;48(9):2450-6. https://doi.org/10.1161/STROKEAHA.117.017320.

\section{Publisher's Note}

Springer Nature remains neutral with regard to jurisdictional claims in published maps and institutional affiliations.

- fast, convenient online submission

- thorough peer review by experienced researchers in your field

- rapid publication on acceptance

- support for research data, including large and complex data types

- gold Open Access which fosters wider collaboration and increased citations

- maximum visibility for your research: over $100 \mathrm{M}$ website views per year

At BMC, research is always in progress.

Learn more biomedcentral.com/submissions 